Boletín de la Sociedad Zoológica del Uruguay, 2020

Vol. 29 (1): 17-23

ISSN: 0255-4402

https://journal.szu.org.uy

\title{
NOTA
}

\section{Epictia albipuncta Burmeister, 1861 (Serpentes: Leptotyphlopidae) first record for the Formosa Province, Argentina}

\author{
José Augusto Ruiz García1, María Esther Tedesco¹, Ornela Sofía Stechina², Jorge Abel Céspedez ${ }^{1}$ \\ ${ }^{1}$ Laboratorio de Herpetología. Facultad de Ciencias Exactas y Naturales y Agrimensura. Universidad Nacional \\ del Nordeste. Av. Libertad 5470 (3400) Corrientes, Argentina. \\ 2Laboratorio de Entomología. Instituto de Medicina Regional. Universidad Nacional del Nordeste. Av. Las \\ Heras 727 (3500) Chaco, Argentina.
}

${ }^{\star}$ Corresponding author: ruizgarciaja @gmail.com

\begin{abstract}
Epictia albipuncta Burmeister, 1861 is a small-sized snake distributed in Argentina, Brasil, Bolivia, Paraguay and Perú. In this study, we report the first record of $E$. albipuncta from Formosa province in the Eastern Chaco District. Our record extends the geographic distribution in $193 \mathrm{~km}$ northeast from the nearest previously known locality Presidencia Roque Sáenz Peña, Chaco province.
\end{abstract}

Keywords: Distribution, Scolecophidia, Snake

\section{RESUMEN}

Epictia albipuncta Burmeister, 1861 (Serpentes: Leptotyphlopidae) primer registro para la provincia de Formosa, Argentina. Epictia albipuncta Burmeister, 1861 es una serpiente de pequeño tamaño que se distribuye en Argentina, Brasil, Bolivia, Paraguay y Perú. En este estudio, reportamos el primer registro de $E$. albipuncta para la provincia de Formosa en el Distrito Chaqueño Oriental. Nuestro registro extiende la distribución geográfica en $193 \mathrm{~km}$ al noreste de la localidad más cercana previamente conocida Presidencia Roque Sáenz Peña en la provincia del Chaco.

Palabras clave: Distribución, Scolecophidia, Serpiente

The genus Epictia (Gray 1845) includes 43 species of small-sized blind snakes distributed from southern Mexico, through Central America (including Caribbean islands), to Argentina and Uruguay (Adalsteinsson et al., 2009; Uetz et al., 2019), and five of them were recorded in Argentina: E. albipuncta, E. australis, E. munoai, E. striatula and E. vellardi (Giraudo et al., 2012). Epictia albipuncta is distributed in Argentina, Brasil, Bolivia, Paraguay and Perú (Cei, 1993; Carrillo de Espinoza \& Icochea, 1995; Kretzschmar, 2006; Cacciali, 2009). In Argentina, this species has a wide distribution, and it was reported in the provinces of Buenos Aires, Catamarca, Chaco, Córdoba, Corrientes, Entre Ríos, Jujuy, Mendoza, Misiones, Neuquén, Salta, Santa Fe, San Juan, Santiago del Estero and Tucumán (Kretzschmar, 2006; Acosta et al., 2017).

In this study, we report the first record of the $E$. albipuncta in the Eastern Chaco District in Formosa province, Argentina.

During a herpetological sampling on $25^{\text {th }}$ October 2010 at 11:20 h we collected a single individual of $E$. albipuncta in Colonia Napenay, Patiño department, Formosa province (25010'33"S; 59041'41"W, WGS84, 106 masl.; Fig. 1). The individual was found under planks of woods partially buried in sandy soil (Fig. 2), the collecting site is located in the Eastern Chaco District, characterized by a forest of Schinopsis balansae and Prosopis sp. (Cabrera \& Willink, 1980).

The specimen was euthanized according to Guidelines for the Euthanasia of Animals (Leary et al., 2013 ), fixed in $10 \%$ formalin solution, and preserved in $70 \%$ ethanol. The snake was deposited at the herpetological collection of the Universidad Nacional del Nordeste, Corrientes (UNNEC 13366). The external morphology of the specimen was analyzed and as Epictia albipuncta following the diagnostic characters indicated by Kretzschmar et al. (2006) and Francisco et al. (2012). Photographs of the specimen were taken with a Leica EZ4 stereoscopic microscope.

The examined specimen corresponds to an adult individual, snout-vent length $163 \mathrm{~mm}$, tail length 10.43 $\mathrm{mm}$. The pholodiosis was recorded as follows: rostral scale hexagonal contacting nasal scale laterally and frontal scale dorsally, with a white blotch covering most of the surface, prefrontal scale subpentagonal and frontal scale hexagonal, interparietal and 


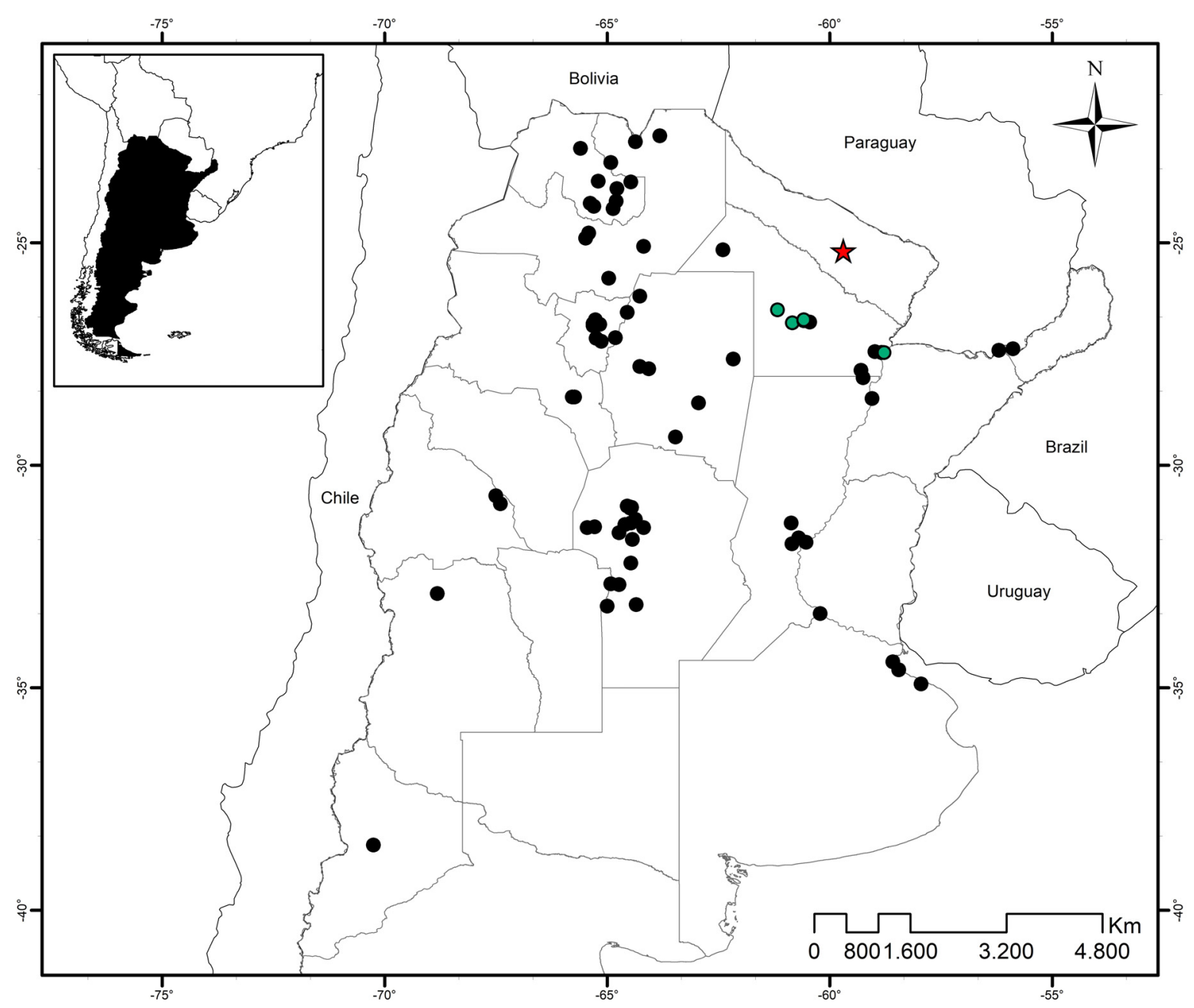

Fig. 1. Map showing known distribution for Epictia albipuncta in Argentina. The red star indicate the new record in Colonia Napenay, department of Patiño, Formosa province. Black circle indicate previous records and green circle records of this study.

interoccipital scales are larger than frontal scale, parietals and occipitals scales oriented transversely, parietals slightly longer than occipitals, nasal scale completely divided, supranasal larger than infranasal scale, ellipsoid nostril located in infranasal scale, ocular scale hexagonal, supraocular scale large and pentagonal, two supralabial scales, anterior supralabial scale is more narrow than posterior infralabial and not contact supraocular scale (Fig. 3A and B), and four infralabial scales, cloacal shield semilunate. The specimen has 258 middorsal scales, 240 ventral scales, 14-14-14 scales rows around body, 20 subcaudals and 12 scales around the middle of the tail. The dorsal surface is background light cream with seven dark longitudinal lines, striped pattern with dark brown triangular blotches (Fig. 3C), ventral surface cream light with seven brown longitudinal lines, caudal spine and last four ventral subcaudals scales white (Fig. 3D).

Based on the locality records of Álvarez et al. (2002; 2003); Kacoliris et al. (2006); Kretzschmar et al. (2006); Acosta et al. (2017) and records from the UNNEC herpetological collection (Table 1) for $E$. albipuncta in Argentina, we extend the known geographic distribution of the species around $193 \mathrm{Km}$ in a straight-line northeast from its nearest previously known locality Presidencia Roque Sáenz Peña, Comandante Fernández department, Chaco province.

The records of leptotyphlopids in Formosa province are scarce since only two species have been previously cited: Epictia vellardi and Rena unguirostris (Laurent, 1984; Cei, 1993). With the 

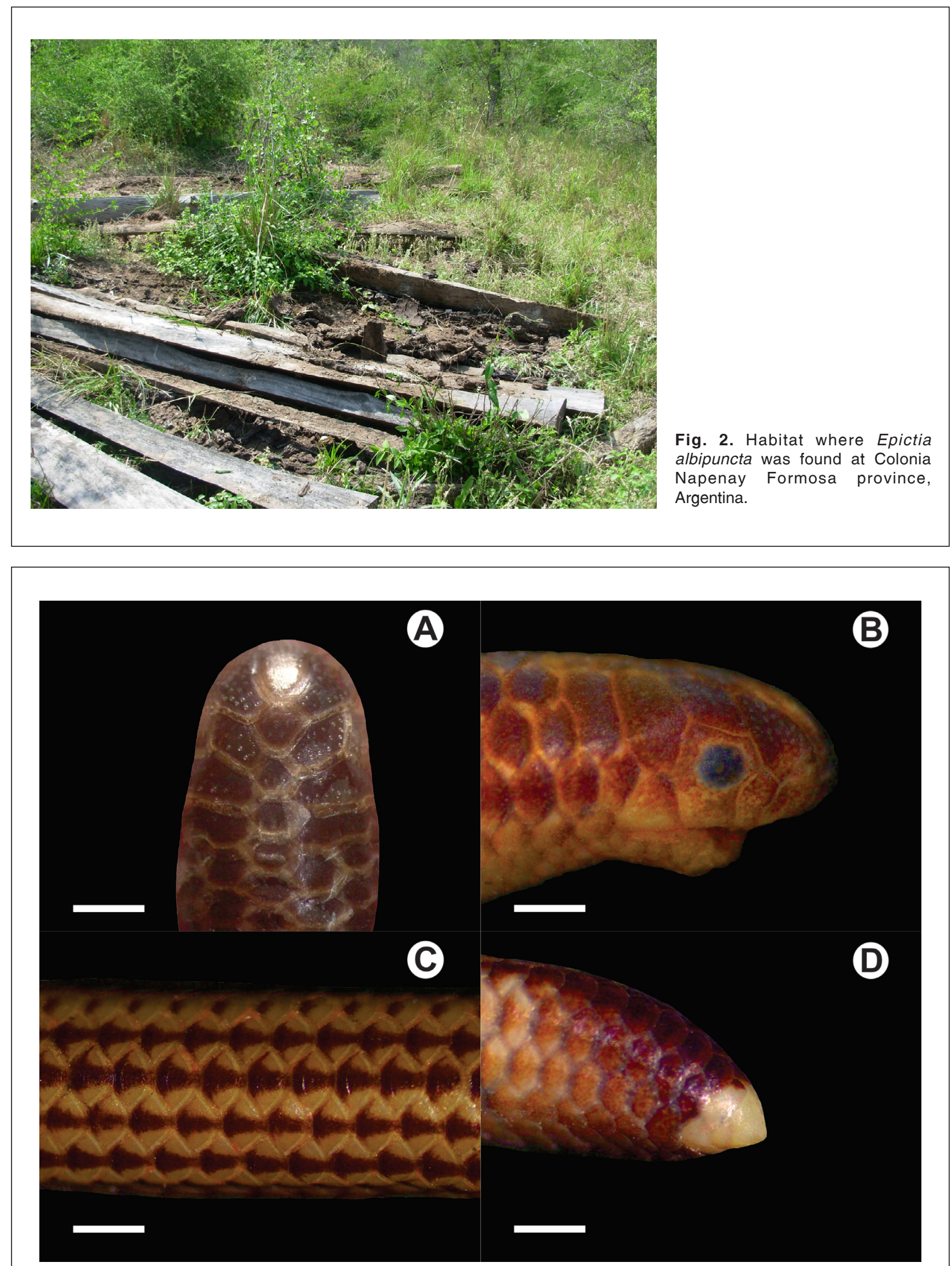

Fig. 3. Epictia albipuncta (UNNEC 13366). A. Dorsal head scale. B. Lateral head scale. C. Striped dorsal pattern with triangular blotches. D. Ventral view of tail. Scale: $1 \mathrm{~mm}$. 
Table 1. Locality records of Epictia albipuncta in Argentina.

\begin{tabular}{|c|c|c|c|c|c|}
\hline \multirow[b]{2}{*}{ Province } & \multirow[b]{2}{*}{ Locality } & \multirow[b]{2}{*}{ Voucher } & \multicolumn{2}{|c|}{ Geographic coordinates } & \multirow[b]{2}{*}{ Reference } \\
\hline & & & Latitude & Longitude & \\
\hline \multirow[t]{5}{*}{ Buenos Aires } & Bosque de la Plata & MLP JW 524 & $34^{\circ} 55^{\prime}$ & $57^{\circ} 57^{\prime}$ & Kretzschmar 2006 \\
\hline & Capital Federal & $\begin{array}{l}\text { MACN ex CENAI } \\
2059,3305\end{array}$ & $34^{\circ} 36^{\prime}$ & $58^{\circ} 27^{\prime}$ & Kretzschmar 2006 \\
\hline & Delta & MACN 1562 & $34^{\circ} 25^{\prime}$ & $58^{\circ} 35^{\prime}$ & Kretzschmar 2006 \\
\hline & Capital Federal & MACN 34575 & $34^{\circ} 36^{\prime}$ & $58^{\circ} 27^{\prime}$ & Kretzschmar 2006 \\
\hline & San Nicolás & $\begin{array}{l}\text { MLP JW 1611, } \\
1612\end{array}$ & $33^{\circ} 20^{\prime}$ & $60^{\circ} 13^{\prime}$ & Kretzschmar 2006 \\
\hline \multirow[t]{3}{*}{ Catamarca } & Catamarca & $\begin{array}{l}\text { MLP JW 274, } \\
275,276\end{array}$ & $28^{\circ} 28^{\prime}$ & $65^{\circ} 47^{\prime}$ & Kretzschmar 2006 \\
\hline & Catamarca & MACN 3070 & $28^{\circ} 28^{\prime}$ & $65^{\circ} 47^{\prime}$ & Kretzschmar 2006 \\
\hline & Sumalao & FML 00933 & $28^{\circ} 28^{\prime}$ & $65^{\circ} 44^{\prime}$ & Kretzschmar 2006 \\
\hline \multirow[t]{8}{*}{ Chaco } & Basail & UNNEC 00318 & $27^{\circ} 52^{\prime}$ & $59^{\circ} 18^{\prime}$ & Kretzschmar 2006 \\
\hline & Campo Largo & $\begin{array}{l}\text { UNNEC 10694, } \\
10695\end{array}$ & $26^{\circ} 48^{\prime} 1^{\prime \prime}$ & $60^{\circ} 50^{\prime} 21^{\prime \prime}$ & This study \\
\hline & Fuerte Esperanza & Distribution map & $25^{\circ} 9^{\prime} 38^{\prime \prime}$ & $61^{\circ} 84^{\prime} 00^{\prime \prime}$ & Kacoliris et al.2006 \\
\hline & La Mascota & UNNEC 9417 & $26^{\circ} 45^{\prime} 19^{\prime \prime}$ & $60^{\circ} 34^{\prime} 54^{\prime \prime}$ & This study \\
\hline & Napenay & UNNEC 008147 & $26^{\circ} 43^{\prime} 57^{\prime \prime}$ & $60^{\circ} 34^{\prime} 54^{\prime \prime}$ & This study \\
\hline & Pampa del Infierno & UNNEC 10355 & $26^{\circ} 30^{\prime} 33^{\prime \prime}$ & $61^{\circ} 10^{\prime} 30^{\prime \prime}$ & This study \\
\hline & Resistencia & UNNEC 00227 & $27^{\circ} 27^{\prime}$ & $58^{\circ} 59^{\prime}$ & Álvarez et al. 2002 \\
\hline & Sáenz Peña & MACN 12531 & $26^{\circ} 47^{\prime}$ & $60^{\circ} 27^{\prime}$ & Kretzschmar 2006 \\
\hline \multirow[t]{17}{*}{ Córdoba } & Achiras & $\begin{array}{l}\text { FML 02773, 02776, } \\
02777,06055,06763\end{array}$ & $33^{\circ 10}$ & $65^{\circ} 00^{\prime}$ & Kretzschmar 2006 \\
\hline & Alpa Corral & FML 02774 & $32^{\circ} 41^{\prime}$ & $64^{\circ} 44^{\prime}$ & Kretzschmar 2006 \\
\hline & Bialet Maseé & FML 02767, 02768 & 31018 & $64^{\circ} 28^{\prime}$ & Kretzschmar 2006 \\
\hline & Cabanas & $\begin{array}{l}\text { MLP JW 575, } \\
\text { MACN } 3085 \text { (12538) }\end{array}$ & $31^{01} 3^{\prime}$ & $64^{\circ} 22^{\prime}$ & Kretzschmar 2006 \\
\hline & Chacay, Sierra de Comechingones & MACN 32052 & $32^{\circ} 40^{\prime}$ & $64^{\circ} 55^{\prime}$ & Kretzschmar 2006 \\
\hline & Córdoba & FML 00359 & $31^{\circ} 24^{\prime}$ & $64^{\circ} 11^{\prime}$ & Kretzschmar 2006 \\
\hline & Corral de Felipe, Cabanas & MACN 4529 (19739) & $31^{\circ 1}$ & $64^{\circ} 22^{\prime}$ & Kretzschmar 2006 \\
\hline & Cruz Chica & MACN 4353 (17821) & $30^{\circ} 58^{\prime}$ & $64^{\circ} 29^{\prime}$ & Kretzschmar 2006 \\
\hline & Cruz Grande & MACN 21449 & $30^{\circ} 56^{\prime}$ & $64^{\circ} 30^{\prime}$ & Kretzschmar 2006 \\
\hline & Icho Cruz & FML 01799 & $31^{\circ} 31^{\prime}$ & $64^{\circ} 44^{\prime}$ & Kretzschmar 2006 \\
\hline & La Bolsa, Alta Gracia & MACN ex CENAI 1451 & $31^{\circ} 40^{\prime}$ & $64^{\circ} 26^{\prime}$ & Kretzschmar 2006 \\
\hline & Los Chorrillos & FML 02770 & $30^{\circ} 57^{\prime}$ & $64^{\circ} 27^{\prime}$ & Kretzschmar 2006 \\
\hline & Río Cuarto & FML 02775 & $33^{\circ} 08^{\prime}$ & $64^{\circ} 21^{\prime}$ & Kretzschmar 2006 \\
\hline & San Esteban & FML 00661, 01168 & $30^{\circ} 55^{\prime}$ & $64^{\circ} 33^{\prime}$ & Kretzschmar 2006 \\
\hline & Segunda Usina. EmbalseRío Tercero & FML 02772 & $32^{\circ} 12^{\prime}$ & $64^{\circ} 28^{\prime}$ & Kretzschmar 2006 \\
\hline & $\begin{array}{l}\text { Sierra de Pocho, Entre LasPalmas y } \\
\text { Chancani }\end{array}$ & FML 00753 & $31^{\circ} 23^{\prime}$ & $65^{017}$ & Kretzschmar 2006 \\
\hline & Tanti & FML 02769 & $31^{\circ} 20^{\prime}$ & $64^{\circ} 36^{\prime}$ & Kretzschmar 2006 \\
\hline
\end{tabular}


Table 1. Continued.

\begin{tabular}{|c|c|c|c|c|c|}
\hline \multirow{2}{*}{ Province } & \multirow[b]{2}{*}{ Locality } & \multicolumn{4}{|c|}{ Geographic coordinates } \\
\hline & & Voucher & Latitude & Longitude & Reference \\
\hline \multirow[t]{10}{*}{ Corrientes } & Bella Vista & MLP JW 536 & $28^{\circ} 30^{\prime}$ & $59^{\circ} 03^{\prime}$ & Kretzschmar 2006 \\
\hline & Capital & UNNEC 10473, 11097 & $27^{\circ} 28^{\prime} 7^{\prime \prime}$ & $58^{\circ} 46^{\prime} 54^{\prime \prime}$ & This study \\
\hline & Concepción & Distribution map & $28^{\circ} 23^{\prime}$ & 57052 & Álvarez et al. 2003 \\
\hline & Corrientes (S/D) & ANSP 3297, 3298 & $27^{\circ} 28^{\prime}$ & $588^{\circ} 50^{\prime}$ & Kretzschmar 2006 \\
\hline & Corrientes & FML 13512 & $27^{\circ} 28^{\prime}$ & $58^{\circ} 50^{\prime}$ & Kretzschmar 2006 \\
\hline & Corrientes & UNNEC 213,214,216 & $27^{\circ} 28^{\prime} 7^{\prime \prime}$ & $58^{\circ} 50^{\prime} 22^{\prime \prime}$ & Álvarez et al. 2002 \\
\hline & Mburucuyá & Distribution map & $28^{\circ} 25^{\prime}$ & $57^{\circ} 54^{\prime}$ & Álvarez et al. 2003 \\
\hline & San Miguel & Distribution map & $27^{\circ} 59^{\prime}$ & $57^{\circ} 36^{\prime}$ & Álvarez et al. 2003 \\
\hline & Villa Olivari & Distribution map & $27^{\circ} 38^{\prime}$ & $56^{\circ} 54^{\prime}$ & Álvarez et al. 2003 \\
\hline & Yacareí & UNNEC 00144 & $27^{\circ} 20^{\prime} 16^{\prime \prime}$ & $58^{\circ} 10^{\prime} 46^{\prime \prime}$ & Kretzschmar 2006 \\
\hline Entre Ríos & Paraná & $\begin{array}{l}\text { MAS 4586, 5170, } \\
5171\end{array}$ & $31^{\circ} 44^{\prime}$ & $60^{\circ} 32^{\prime}$ & Kretzschmar 2006 \\
\hline Formosa & Colonia Napenay & UNNEC 13366 & $25^{\circ} 10^{\prime} 33^{\prime \prime}$ & $59^{\circ} 41^{\prime} 41^{\prime \prime}$ & This study \\
\hline \multirow[t]{5}{*}{ Jujuy } & Calilegua & FML 01229 & $23^{\circ} 47^{\prime}$ & $64^{\circ} 47^{\prime}$ & Kretzschmar 2006 \\
\hline & Chalicán & MACN 3027 (12450) & $24^{\circ} 04^{\prime}$ & $64^{\circ} 48^{\prime}$ & Kretzschmar 2006 \\
\hline & Las Capillas (La Capilla) & MACN 3067 & $22^{\circ} 53^{\prime}$ & $65^{\circ} 36^{\prime}$ & Kretzschmar 2006 \\
\hline & San Pedro, Ingenio La Esperanza & FML 01326, 01947 & $24^{0} 14^{\prime}$ & $64^{\circ} 52^{\prime}$ & Kretzschmar 2006 \\
\hline & San S. de Jujuy & MLP JW 277, 278 & $24^{0} 11^{\prime}$ & $65^{0} 18^{\prime}$ & Kretzschmar 2006 \\
\hline \multirow[t]{2}{*}{ Jujuy } & Yala & MACN 25397 & $24^{\circ} 07^{\prime}$ & $65^{\circ} 23^{\prime}$ & Kretzschmar 2006 \\
\hline & Yuto & FML 00268, 01319 & $23^{\circ} 38^{\prime}$ & $64^{\circ} 28^{\prime}$ & Kretzschmar 2006 \\
\hline Mendoza & Mendoza & MAS 4496 & $32^{\circ} 53^{\prime}$ & $68^{\circ} 49^{\prime}$ & Kretzschmar 2006 \\
\hline Misiones & Posadas & CUNaM 256 & $27^{\circ} 23^{\prime}$ & $55^{\circ} 53^{\prime}$ & Kretzschmar 2006 \\
\hline Neuquén & Convuco & MACN 1891 (8647) & $38^{\circ} 32^{\prime}$ & $70^{\circ} 15^{\prime}$ & Kretzschmar 2006 \\
\hline \multirow[t]{9}{*}{ Salta } & Cerrillos, Partido La Candelaria & FML 01421 & $24^{\circ} 54^{\prime}$ & $65^{\circ} 29^{\prime}$ & Kretzschmar 2006 \\
\hline & Finca Pozo Largo & FML 02082, 02311 & $25^{\circ} 05^{\prime}$ & $64^{\circ} 11^{\prime}$ & Kretzschmar 2006 \\
\hline & Finca San Javier & FML 02307 & $25^{\circ} 05^{\prime}$ & $64^{\circ} 11^{\prime}$ & Kretzschmar 2006 \\
\hline & Río Pescado (Aguas Blancas) & FML 00625 & $22^{\circ} 44^{\prime}$ & $64^{\circ} 22^{\prime}$ & Kretzschmar 2006 \\
\hline & Rosario de la Frontera. Finca & MACN 35522 & $25^{\circ} 48^{\prime}$ & $64^{\circ} 58^{\prime}$ & Kretzschmar 2006 \\
\hline & El Duraznito & & & & \\
\hline & Salta & FML 02312 & $24^{\circ} 47^{\prime}$ & $65^{\circ} 25^{\prime}$ & Kretzschmar 2006 \\
\hline & Santa Cruz & FML 00765 & $23^{\circ} 12^{\prime}$ & $64^{\circ} 55^{\prime}$ & Kretzschmar 2006 \\
\hline & Vespucio & MACN ex CENAI 2184 & $22^{\circ} 36^{\prime}$ & $63^{\circ} 49^{\prime}$ & Kretzschmar 2006 \\
\hline \multirow[t]{2}{*}{ San Juan } & Las Tumanas & UNSJ 2547 & $30^{\circ} 52^{\prime} 00^{\prime \prime}$ & $67^{\circ} 24^{\prime} 00^{\prime \prime}$ & Acosta et al. 2017 \\
\hline & La Majadita & UNSJ 2548 & $30^{\circ} 41^{\prime} 12^{\prime \prime}$ & 67030'13”' & Acosta et al. 2017 \\
\hline \multirow[t]{4}{*}{ Santa Fe } & Florencia & UNNEC 06797 & $28^{\circ} 02^{\prime}$ & $59^{\circ} 15^{\prime}$ & Kretzschmar 2006 \\
\hline & La Capital & $\begin{array}{l}\text { FML 01370, MACN } \\
28782\end{array}$ & 31018 & $60^{\circ} 52^{\prime}$ & Kretzschmar 2006 \\
\hline & Santa Fe, Km 11 & MACN 1243 (7035) & $31^{\circ} 38^{\prime}$ & $60^{\circ} 42^{\prime}$ & Kretzschmar 2006 \\
\hline & Sauce Viejo & FML 06604 & $31^{\circ} 46^{\prime}$ & $60^{\circ} 51^{\prime}$ & Kretzschmar 2006 \\
\hline
\end{tabular}


Table 1. Continued.

\begin{tabular}{|c|c|c|c|c|c|}
\hline & \multicolumn{4}{|c|}{ Geographic coordinates } \\
\hline Province & Locality & Voucher & Latitude & Longitude & Reference \\
\hline \multirow{6}{*}{$\begin{array}{l}\text { Santiago del } \\
\text { Estero }\end{array}$} & Nueva Esperanza & FML 02130 & $26^{0} 12^{\prime}$ & $64^{\circ} 16^{\prime}$ & Kretzschmar 2006 \\
\hline & Santiago del Estero & $\begin{array}{l}\text { MACN ex CENAI } \\
2060,2061,2062\end{array}$ & $27^{\circ} 47^{\prime}$ & $64^{\circ} 16^{\prime}$ & Kretzschmar 2006 \\
\hline & Beltrán & $\begin{array}{l}\text { MLP JW 526, 339, } \\
448\end{array}$ & $27^{0} 50$ & $64^{\circ} 04^{\prime}$ & Kretzschmar 2006 \\
\hline & Colonia Dora & $\begin{array}{l}\text { MACN ex CENAI } \\
2113,2114,2115\end{array}$ & $28^{\circ} 36^{\prime}$ & $62^{\circ} 57^{\prime}$ & Kretzschmar 2006 \\
\hline & Girardet & MLP JW 099 & $27^{\circ} 37^{\prime}$ & $62^{\circ} 10^{\prime}$ & Kretzschmar 2006 \\
\hline & Sumampa & MAS 2245 & $29^{\circ} 22^{\prime}$ & $63^{\circ} 28^{\prime}$ & Kretzschmar 2006 \\
\hline \multirow[t]{14}{*}{ Tucumán } & Banda del Río Salí & FML 02472 & $26^{\circ} 50^{\prime}$ & $65^{\circ} 10^{\prime}$ & Kretzschmar 2006 \\
\hline & El Cadillal & $\begin{array}{l}\text { FML 00736, } \\
06057,06073\end{array}$ & $23^{\circ} 37^{\prime}$ & $65^{\circ 12}$ & Kretzschmar 2006 \\
\hline & Garmendia & FML 00965 & $26^{\circ} 34^{\prime}$ & $64^{\circ} 33^{\prime}$ & Kretzschmar 2006 \\
\hline & Ingenio San Pablo & FML 01205 & $26^{\circ} 52^{\prime}$ & $65^{\circ} 19^{\prime}$ & Kretzschmar 2006 \\
\hline & Las Talitas & FML 02440 & $26^{\circ} 49^{\prime}$ & $65^{\circ} 13^{\prime}$ & Kretzschmar 2006 \\
\hline & Mistas & FML 01954 & $27^{0} 13^{\prime}$ & $65^{\circ} 08^{\prime}$ & Kretzschmar 2006 \\
\hline & Orilla del Canal Norte & FML 01600 & $26^{\circ} 44^{\prime}$ & $65^{\circ} 16^{\prime}$ & Kretzschmar 2006 \\
\hline & Río Loro, El Cadillal & FML 01300 & $23^{\circ} 37^{\prime}$ & $65^{\circ 1} 2^{\prime}$ & Kretzschmar 2006 \\
\hline & San Miguel de Tucumán & $\begin{array}{l}\text { MNHN 911, FML 00603, } \\
\text { 00630, 00652, } 00662 \text {, } \\
\text { 00664, 00699, } 00731\end{array}$ & $26^{\circ} 49^{\prime}$ & $65^{\circ} 13^{\prime}$ & Kretzschmar 2006 \\
\hline & San Pablo & FML 01234 & $26^{\circ} 52^{\prime}$ & $65^{\circ} 19^{\prime}$ & Kretzschmar 2006 \\
\hline & Santa Rosa de Leales & FML 00147 & $27^{\circ} 09^{\prime}$ & $65^{\circ} 15^{\prime}$ & Kretzschmar 2006 \\
\hline & Tacanas & FML 00766 & $27^{\circ} 08^{\prime}$ & $64^{\circ} 49^{\prime}$ & Kretzschmar 2006 \\
\hline & Tucumán (S/D) & $\begin{array}{l}\text { MLU IZH-R } 461 \\
\text { (HOLOTIPO) }\end{array}$ & $26^{\circ} 49^{\prime}$ & $65^{\circ} 13^{\prime}$ & Kretzschmar 2006 \\
\hline & Yerba Buena & $\begin{array}{l}\text { FML 00775,00791, } \\
1601\end{array}$ & $26^{\circ} 49^{\prime}$ & $65^{\circ} 19^{\prime}$ & Kretzschmar 2006 \\
\hline
\end{tabular}

record of E. albipuncta, the richness of snakes in the Formosa province amounts to 56 species (Giraudo et al., 2012). Our record completes the distribution gap of the species for northern of Argentina.

\section{ACKNOWLEDGMENTS}

We thank SGCYT-UNNE (Secretaría General de Ciencia y Técnica de la Universidad Nacional del Nordeste) for financial support to the Project (F02008) "Historia Natural de Anfibios y reptiles del Nordeste Argentino."

\section{REFERENCES}

Acosta R., Blanco G.M., Galdeano A.P., Gómez Alés R. \& Acosta J.C. 2017. Primer registro de Epictia albipuncta Burmeister, 1861 (Serpentes: Leptotyphlopidae) en la provincia de San Juan, Argentina. Cuadernos de Herpetología, 31(2):127-128.

Adalsteinsson S.A., Branch W.R., Trape S., Vitt L.J. \& Blair Hedges S. 2009. Molecular phylogeny, classification, and biogeography of snakes of the Family Leptotyphlopidae (Reptilia, Squamata). Zootaxa, 2244 (1):1-50.

Álvarez B.B., Aguirre R., Céspedez J., Hernando A. \& 
Tedesco M.E. 2003. Herpetofauna del Iberá. En: Álvarez B.B. (Ed.) Fauna del Iberá. EUDENE, pp. 99-178. Editorial Universitaria de la Universidad Nacional del Nordeste, Corrientes.

Álvarez B.B., Aguirre R.H., Céspedez J.A., Hernando A., Tedesco E. \& Orfeo O. 2002. Atlas de Anfibios y Reptiles de las Provincias de Corrientes, Chaco y Formosa (Argentina) I (Anuros, Cecílidos, Saurios, Anfisbénidos y Serpientes). EUDENE, Editorial Universitaria de la Universidad Nacional del Nordeste, Corrientes. $160 \mathrm{pp}$.

Cabrera A.L. \& Willink A. 1980. Biogeografía de América Latina. OEA, Serie Biología, Monografía, 13: 1-122.

Cacciali P. 2009. Guía para la identificación de 60 Serpientes del Paraguay. Asociación Guyra Paraguay, Asunción. 218 pp.

Carrillo de Espinoza N. \& Icochea J. 1995. Lista taxonómica preliminar de los reptiles vivientes del Perú. Publicaciones del Museo de Historia Natural Universidad Nacional Mayor de San Marcos, 49:1-27.

Cei J.M. 1993. Reptiles del noroeste, nordeste y este de la Argentina. Bolletino Museo Regionale di Scienze Naturali, Torino, Monografie 4: 1-949.

Francisco B.C.S., Pinto R.R. \& Fernandes D.S. 2012 Taxonomy of Epictia munoai (Orejas-Miranda, 1961) (Squamata: Serpentes: Leptotyphlopidae). Zootaxa, 3512 (1): 42-52.

Giraudo A., Arzamendia V., Bellini G.P., Bessa C.A.,
Calamante C.C., Cardozo G., Chiaraviglio M., Costanzo M.B., Etchepare E., Di Cola V., Di Pietro D., Kretzschmar S., Palomas S., Nenda S.J., Rivera P., Rodríguez M.E., Scrocchi G.J. \& Williams J.D. 2012. Categorización del estado de conservación de las Serpientes de la República Argentina. Cuadernos de Herpetologia, 26 (Supl. 1): 303-326.

Kacoliris F.P., Berkunsky I. \& Williams J. 2006. Herpetofauna of the Argentinean impenetrable Great Chaco. Phyllomedusa, 5 (2): 149-157.

Kretzschmar S. 2006. Revisión histórica y redescripción de Leptotyphlops albipunctus (Serpentes: Leptotyphlopidae).Cuadernos de Herpetología, 19 (2):43-56.

Laurent R.F. 1984. El género Leptotyphlops en la colección de la Fundación Miguel Lillo. Acta Zoológica Lilloana, 38 (1): 29-34.

Leary S., Underwood W., Anthony R., Cartner S., Corey D., Grandin T., Greenacre C.B., GwaltneyBran S., McCrackin M.A., Meyer R., Miller D., Shearer J. \& Yanong R. 2013. AVMA Guidelines for the Euthanasia of Animals, 2013 Edition. American Veterinary Medical Association, Schaumburg, IL.

Uetz P., Freed P. \& Hosek J. 2019. The Reptile Database. http://www.reptile-database.org. Accesed on: 2019-05-08.

Fecha de Recepción: 17 de octubre de 2019 Fecha de Aceptación: 09 de julio de 2020 